\title{
Apolipoproteins A-I, B, and C-III and Obesity in Young Adult Cherokee
}

\author{
Wenyu Wang, ${ }^{1}$ Piers Blackett, ${ }^{2}$ Sohail Khan, ${ }^{3}$ and Elisa Lee ${ }^{1}$ \\ ${ }^{1}$ Center for American Indian Health Research, College of Public Health, University of Oklahoma Health Sciences Center, \\ Oklahoma City, OK 73190, USA \\ ${ }^{2}$ Section of Diabetes and Endocrinology, Department of Pediatrics, Harold Hamm Diabetes Center, \\ University of Oklahoma Health Sciences Center, Oklahoma City, OK 73104, USA \\ ${ }^{3}$ The Cherokee Nation, P.O. Box 948, Tahlequah, OK 74465, USA \\ Correspondence should be addressed to Piers Blackett; piersblackett@att.net
}

Received 8 February 2017; Accepted 20 March 2017; Published 3 April 2017

Academic Editor: Gerd Schmitz

Copyright (c) 2017 Wenyu Wang et al. This is an open access article distributed under the Creative Commons Attribution License, which permits unrestricted use, distribution, and reproduction in any medium, provided the original work is properly cited.

Since young adult Cherokee are at increased risk for both diabetes and cardiovascular disease, we assessed association of apolipoproteins (A-I, B, and C-III in non-HDL and HDL) with obesity and related risk factors. Obese participants (BMI $\geq 30$ ) aged 20-40 years $(n=476)$ were studied. Metabolically healthy obese $(\mathrm{MHO})$ individuals were defined as not having any of four components of the ATP-III metabolic syndrome after exclusion of waist circumference, and obese participants not being MHO were defined as metabolically abnormal obese (MAO). Associations were evaluated by correlation and regression modeling. Obesity measures, blood pressure, insulin resistance, lipids, and apolipoproteins were significantly different between groups except for total cholesterol, LDL-C, and HDL-apoC-III. Apolipoproteins were not correlated with obesity measures with the exception of apoA-I with waist and the waist : height ratio. In a logistic regression model apoA-I and the apoB : apoA-I ratio were significantly selected for identifying those being $\mathrm{MHO}$, and the result $(C$-statistic $=0.902)$ indicated that apoA-I and the apoB : apoA-I ratio can be used to identify a subgroup of obese individuals with a significantly less atherogenic lipid and apolipoprotein profile, particularly in obese Cherokee men in whom MHO is more likely.

\section{Introduction}

Since obesity predicts atherosclerotic cardiovascular disease (ACVD), it has significant worldwide health and economic implications [1]. This is particularly true in the Cherokee and other American Indian populations [2] in whom obesity is associated with the metabolic syndrome, which often precedes type 2 diabetes (T2D) [3]. Consequently it has become important to study association of obesity with apolipoproteins, since obesity-associated changes in lipid transport precede and predict subsequent insulin resistance and ultimately the development of ACVD and T2D [4, 5]. Therefore, we selected apolipoproteins known to predict atherosclerosis for study. We also proposed that the obese participants could be classified as two distinct groups based on the presence of metabolic complications including dyslipidemia [6] and that apolipoprotein levels might serve to identify differences between the metabolically healthy obese (MHO) and metabolically abnormal obese (MAO) groups.

Apolipoprotein B (apoB) represents the total number of apoB-containing lipoproteins [7] and is considered to be superior to LDL-C and non-HDL-C in predicting cardiovascular disease [8], whereas apolipoprotein A-I (apoA-I) has a known inverse association and low levels are associated with increased body mass index (BMI) [9]. Furthermore, the ratio of apoB to apoA-I (B:A-I ratio), representing the combination of two atherogenic processes, is an even stronger predictor [10]. Apolipoprotein C-III (apoC-III) is secreted with VLDL and becomes distributed among circulating lipoproteins [11] conferring harmful properties resulting in ACVD $[3,12]$. LDL particles containing apoC-III are more 
atherogenic than particles without apoC-III [13] and apoC-III on non-HDL lipoprotein particles independently predicted recurrent coronary events [14] and progression of carotid intima-media thickness during treatment [15]. Following hepatic secretion of apoC-III as VLDL, its subsequent distribution on HDL particles may also be harmful, since HDLapoC-III predicted angiographic progression of atherosclerosis in bypass grafts [16] and more recently HDL-apoC-III has been identified as a proatherogenic HDL subtype with loss of its anti-inflammatory properties [16].

Genetic deficiency [17] and targeted gene disruption [18] of apoC-III have been shown to be associated with protection from atherosclerosis [17]. However, the relative role of apoCIII's distribution on lipoproteins remains uncertain [19], and preliminary evidence suggests that obesity may play a central role in determining apoC-III levels, lipoprotein distribution, and clinical outcomes [20]. Consequently this study and analysis were done to examine association of obesity with apoB, apoA-I, and apoC-III content of both non-HDL and HDL.

\section{Methods}

With collaboration of the Cherokee Nation of Oklahoma, adults aged 20-40 years in the Cherokee Diabetes Study cohort residing in a 5-county area in northeastern Oklahoma participated in the study $(n=1051)$. Of this group $477(45 \%)$ were obese, defined as having a BMI greater than or equal to 30. Nondiabetic participants were excluded according to American Diabetes Association criteria for fasting plasma glucose (FPG) defined as being greater than or equal to $126 \mathrm{mg} / \mathrm{dl}$ or being on medications for diabetes. Informed consent was obtained from each subject or his/her legal guardian, following approval of the Institutional Review Boards of the University of Oklahoma Health Sciences Center and the Cherokee Nation.

After obtaining clinical measurements, fasting blood specimens were collected for determining FPG, insulin, lipids, and apolipoproteins.

2.1. Lipids and Apolipoproteins. An Abbott VP-Super System automatic analyzer and commercial reagents were used to determine levels of glucose, cholesterol (Boehringer, Mannheim, Germany), and triglyceride (Miles Inc., Tarrytown, NJ) by enzymatic methodology. HDL-C was measured using the heparin-manganese precipitation procedure of the Lipid Research Clinics program and LDL-C was calculated by the Friedewald formula. ApoA-I, apoB, and apoC-III were determined by previously validated electroimmunoassays [21-23]. The apoC-III concentrations in whole plasma and heparin-manganese supernatant were determined by separate assays. ApoC-III in the precipitate was calculated by subtracting the supernatant value from the total plasma apoC-III.

2.2. Glucose and Insulin. Fasting insulin levels were determined in a National Institutes of Health core laboratory at the Endocrinology Department at the University of Chicago.
Insulin was measured in serum samples using a competitive double antibody radioimmunoassay and glucose by an automated method using glucose oxidase (Alfa Wassermann, Inc., West Caldwell, NJ). The homeostasis index (HOMA-IR) was computed from the product of insulin $(\mathrm{lU} / \mathrm{mL})$ and glucose ( $\mathrm{mmol} / \mathrm{L})$ divided by 22.5 .

\subsection{Blood Pressure, Waist Circumference, and Height Measure-} ments. Three consecutive measurements of systolic blood pressure (SBP) and diastolic blood pressure (DBP) were performed on the right arm using a Baum mercury sphygmomanometer (W.A. Baum Co., Copiague, NY), and the average of the second and third measurements was recorded. The average of duplicate measures of the waist circumference and height was obtained using a nonstretchable linen tape for the waist circumference and a wall-mounted calibrated stadiometer for the height measurements.

2.4. Statistical Analysis. Means and standard deviations were estimated on all measurements on obese participants aged 20-40 years. Obesity was defined as having a BMI $\geq 30$. Participants were separated by subgroups of metabolically healthy obese (MHO) individuals, defined as having none of four National Cholesterol Education Program (NCEP) metabolic syndrome components after excluding waist circumference. Metabolically abnormal obese (MAO) individuals had at least one of the four criteria. The mean difference of a variable between the two subgroups was calculated after adjusting for age and gender. Logarithmic transformation was used if a variable was not symmetrically distributed such as triglyceride. Spearman partial correlation was done between variables after adjusting for age and gender. To explore odds that an obese participant is classified as being $\mathrm{MHO}$ and distinguish from being $\mathrm{MAO}$, logistic regression was used to select significant determinants with adjustment for age and gender. Statistical analyses were conducted with SAS (version 9.4), and a $P<0.05$ was considered as significant.

\section{Results}

Measures of obesity (BMI, waist circumference, and waist to height ratio), blood pressure (systolic and diastolic), glucose homeostasis (fasting glucose, insulin, and the homeostasis index), lipids, and apolipoproteins were different between individuals being $\mathrm{MHO}$ and MAO with the exception of TC, LDL-C, and HDL-apoC-III (Table 1).

Significant correlations were observed between waist and waist to height ratio with apoA-I; diastolic blood pressure with HDL-apoC-III; and insulin and HOMA-IR with apoB, apoA-I, apoB:apoA-I ratio, and non-HDLapoC-III (Table 2). Lipids showed expected correlations with apolipoproteins with exception of triglyceride with apoA-I.

In the logistic regression model for odds or probability of an obese individual being $\mathrm{MHO}$, apoA-I and apoB : apoAI ratio were significantly selected among apoB, apoA-I, apoB : apoA-I, LpA-I, LpA-I : A-II, non-HDL-apoC-III, and HDL-apoC-III into the model for identifying those with 
TABLE 1: Mean values, standard deviations, and differences $(P<0.05$, bold type) between metabolically healthy obese (MHO) individuals, defined as those who are obese without any of four abnormal National Cholesterol Education Program (NCEP) metabolic syndrome components after excluding waist circumference, and metabolically abnormal obese (MAO) individuals defined as having one or more of the four abnormal criteria. Obesity was defined as body mass index (BMI) $\geq 30$ for young adult Cherokee aged 20-40.

\begin{tabular}{|c|c|c|c|c|c|}
\hline & \multicolumn{2}{|c|}{$\operatorname{MHO}(n=41)$} & \multicolumn{2}{|c|}{ MAO $(n=435)$} & \multirow{2}{*}{$P$} \\
\hline & Mean & STD & Mean & STD & \\
\hline \multicolumn{6}{|l|}{ Obesity } \\
\hline Male & $56.1 \%$ & & $41.1 \%$ & & 0.0643 \\
\hline BMI & 33.87 & 3.87 & 36.81 & 6.90 & 0.0096 \\
\hline Waist (cms) & 102.95 & 10.78 & 109.43 & 13.54 & 0.0006 \\
\hline Waist : height & 0.62 & 0.05 & 0.66 & 0.09 & 0.0022 \\
\hline \multicolumn{6}{|c|}{ Blood pressure (mm Hg) } \\
\hline SBP & 118.59 & 7.57 & 122.88 & 13.01 & 0.0009 \\
\hline DBP & 75.55 & 6.9676 & 79.41 & 9.6968 & 0.0007 \\
\hline \multicolumn{6}{|l|}{ Insulin resistance } \\
\hline FPG (mmol/L) & 4.55 & 0.55 & 4.85 & 0.62 & 0.0047 \\
\hline Insulin $(\mu \mathrm{U} / \mathrm{ml})$ & 114.60 & 62.45 & 181.24 & 116.0 & 0.0002 \\
\hline $\log ($ Insulin $)$ & 4.59 & 0.57 & 5.03 & 0.57 & $<0.0001$ \\
\hline HOMA-IR & 3.88 & 2.23 & 6.58 & 4.51 & 0.0001 \\
\hline \multicolumn{6}{|l|}{ Lipids $(\mathrm{mmol} / \mathrm{L})$} \\
\hline $\mathrm{TC}$ & 4.37 & 0.87 & 4.42 & 0.89 & 0.8534 \\
\hline TG & 0.87 & 0.36 & 1.42 & 0.80 & $<0.0001$ \\
\hline $\log (\mathrm{TG})$ & 4.25 & 0.4570 & 4.69 & 0.5366 & $<0.0001$ \\
\hline LDL-C & 2.65 & 0.81 & 2.83 & 0.78 & 0.2249 \\
\hline HDL-C & 1.31 & 0.19 & 0.95 & 0.22 & $<0.0001$ \\
\hline Non-HDL-C & 3.05 & 0.88 & 3.47 & 0.88 & 0.0040 \\
\hline \multicolumn{6}{|c|}{ Apolipoproteins $(\mu \mathrm{mol} / \mathrm{L})$} \\
\hline ApoB & 1.56 & 0.37 & 1.75 & 0.40 & 0.0023 \\
\hline ApoA-I & 2.56 & 0.31 & 2.15 & 0.32 & $<0.0001$ \\
\hline ApoB : apoA-I & 0.62 & 0.1542 & 0.83 & 0.2046 & $<0.0001$ \\
\hline LpA-1 & 0.57 & 0.08 & 0.47 & 0.09 & $<0.0001$ \\
\hline LpA-1: A-II & 1.99 & 0.24 & 1.68 & 0.24 & $<0.0001$ \\
\hline Non-HDL-apoC-III & 0.06 & 0.03 & 0.09 & 0.05 & 0.0001 \\
\hline HDL-apoC-III & 0.09 & 0.02 & 0.09 & 0.02 & 0.4069 \\
\hline
\end{tabular}

MHO among all the obese participants (Table 3). ApoA-I has a positive association while apoB : apoA-I ratio has a strong negative association with the odds of an obese individual being MHO. Assuming that the other variables in the model are the same, males had a more than four times chance of being MHO than females; and for one standard deviation higher apoA-I or apoB : apoA-I, the chance of being $\mathrm{MHO}$ is increased by 2.57 times or reduced by $69 \%$, respectively (Table 3).

Figure 1 shows the receiver operating characteristics (ROC) curve derived from the logistic regression. The area under the curve ( $C$-statistic) was 0.902 , implying high discrimination ability of the model and consequently of both apoA-I and the apoB : apoA-I ratio in identifying those being MHO among all the obese participants. For a sensitivity of $90 \%$ the respective highest specificity is $81 \%$ and the corresponding cutoff probability is 0.0855 from the ROC curve (Figure 1).

\section{Discussion}

Paradoxically low non-HDL-apoC-III with relatively less atherogenic lipids and lipoproteins, resembling "metabolically healthy" obesity [6] with reduced cardiovascular risk [24] was present in a young adult Cherokee population. This group had reduced adiposity and less insulin resistance as has been observed in cross-sectional studies $[25,26]$. This distinct entity may represent a transient phase in a sequence of worsening insulin resistance, a concept supported by the Atherosclerosis Risk in Communities (ARIC) study showing that risk factors increase after three years of follow-up when compared to a nonobese group [27]. Therefore, prescription of weight management for all obese patients, including the "metabolically healthy," would be prudent to avoid atherogenic risk factor progression.

Insulin resistance, measured as the homeostasis index (HOMA-IR) and the fasting insulin level, correlated with 
TABLE 2: Spearman partial correlation between selected variables after adjusting for age and gender for obese young adult Cherokee aged $20-40(N=476)$.

\begin{tabular}{|c|c|c|c|c|c|c|c|c|c|c|}
\hline & \multicolumn{2}{|c|}{ ApoB } & \multicolumn{2}{|c|}{ ApoA-I } & \multicolumn{2}{|c|}{ ApoB : apoA-I } & \multicolumn{2}{|c|}{ Non-HDL-apoC-III } & \multicolumn{2}{|c|}{ HDL-apoC-III } \\
\hline & $R$ & $P$ & $R$ & $P$ & $R$ & $P$ & $R$ & $P$ & $R$ & $P$ \\
\hline \multicolumn{11}{|l|}{ Obesity } \\
\hline BMI & -0.012 & 0.803 & -0.085 & 0.074 & 0.047 & 0.326 & 0.052 & 0.275 & -0.058 & 0.217 \\
\hline Waist & -0.029 & 0.542 & -0.123 & 0.009 & 0.044 & 0.349 & 0.070 & 0.140 & -0.062 & 0.189 \\
\hline Waist : height & -0.015 & 0.755 & -0.109 & 0.021 & 0.056 & 0.241 & 0.049 & 0.304 & -0.077 & 0.106 \\
\hline \multicolumn{11}{|l|}{ Blood pressure } \\
\hline SBP & 0.037 & 0.429 & 0.062 & 0.192 & -0.008 & 0.863 & -0.003 & 0.956 & 0.038 & 0.423 \\
\hline DBP & 0.049 & 0.296 & 0.011 & 0.824 & 0.035 & 0.464 & 0.038 & 0.428 & 0.099 & 0.036 \\
\hline \multicolumn{11}{|c|}{ Insulin resistance } \\
\hline FPG & 0.068 & 0.154 & -0.037 & 0.441 & 0.066 & 0.164 & 0.027 & 0.568 & 0.024 & 0.610 \\
\hline HOMA-IR & 0.112 & 0.018 & -0.106 & 0.025 & 0.173 & 0.000 & 0.183 & 0.000 & 0.037 & 0.441 \\
\hline Insulin & 0.107 & 0.023 & -0.102 & 0.030 & 0.172 & 0.000 & 0.190 & 0.000 & 0.035 & 0.463 \\
\hline \multicolumn{11}{|l|}{ Lipids } \\
\hline $\mathrm{TC}$ & 0.814 & $<0.001$ & 0.340 & $<0.001$ & 0.505 & $<0.001$ & 0.510 & $<0.001$ & 0.369 & $<0.001$ \\
\hline LDL-C & 0.725 & $<0.001$ & 0.178 & 0.000 & 0.528 & $<0.001$ & 0.299 & $<0.001$ & 0.163 & 0.001 \\
\hline HDL-C & -0.143 & 0.002 & 0.759 & $<0.001$ & -0.562 & $<0.001$ & -0.299 & $<0.001$ & 0.192 & $<0.001$ \\
\hline Non-HDL-C & 0.862 & $<0.001$ & 0.132 & 0.005 & 0.672 & $<0.001$ & 0.588 & $<0.001$ & 0.316 & $<0.001$ \\
\hline TG & 0.602 & $<0.001$ & -0.090 & 0.057 & 0.574 & $<0.001$ & 0.886 & $<0.001$ & 0.449 & $<0.001$ \\
\hline
\end{tabular}

$P, P$ value for Spearman partial correlation after adjusting for age and gender, and significant values $(P<0.05)$ are in bold font; $R$, Spearman partial correlation.

TABLE 3: Logistic regression model for odds or probability of an obese participant being metabolically healthy obese (MHO).

\begin{tabular}{|c|c|c|c|c|c|c|}
\hline Variable & Estimate & SE & $P$ & Unit* & OR & $95 \%$ CI \\
\hline Intercept & -4.245 & 1.888 & 0.0246 & & & \\
\hline Age & -0.049 & 0.036 & 0.1770 & 5 & 0.78 & $0.54,1.11$ \\
\hline Male versus female & 1.433 & 0.401 & 0.0004 & & 4.19 & $1.94,9.46$ \\
\hline ApoA-I & 0.055 & 0.012 & $<0.0001$ & 17.19 & 2.57 & $1.75,3.90$ \\
\hline ApoB : apoA-I & -5.594 & 1.361 & $<0.0001$ & 0.21 & 0.31 & $0.17,0.53$ \\
\hline$C$-statistic & 0.902 & & & & & \\
\hline
\end{tabular}

${ }^{*}$ One standard deviation used for apoA-I and apoB : apoA-I.

$\mathrm{CI}$, confidence interval; $C$-statistic, the area under the receiver operating characteristic curve; Estimate, estimated coefficient; OR, odds ratio; $P, P$ value; SE, standard error.

apoB, with the apoB: apoA-I ratio, and inversely with apoAI supporting known association of insulin resistance with lipoprotein transport [12]. Correlations of HOMA-IR and fasting insulin with non-HDL-apoC-III but not with HDLapoC-III can be accounted for by an effect of insulin resistance on VLDL prior to apoC-III's transfer to HDL during lipolysis [28]. Since apoC-III has a known role in cardiovascular disease $[14,20]$ it may be a marker for atherosclerosis associated with obesity. Non-HDL-apoC-III is an independent predictor of atherosclerosis [15], and LDL containing both apoB and apoC-III is more atherogenic than LDL containing apoB alone [13], supporting an obesityassociated increase in risk attributable to apoC-III. Its atherogenic properties are further supported by adverse effects on the arterial wall including enhanced LDL binding to biglycan [29] and proinflammatory effects via nuclear factor kB-mediated VCAM-1 expression and monocyte adhesion [30]. We have previously shown that non-HDL-apoC-III is proportionate to the number of metabolic syndrome criteria
[3] supporting contribution to cardiovascular risk in obesity, a proposed contributor to the syndrome [4]. Furthermore, hepatic insulin resistance influences apoC-III transcription $[31,32]$ beginning in childhood [32]. Also the apoC-III promotor contains a carbohydrate response element suggesting that dietary glucose and possibly saturated fatty acids increase transcription $[33,34]$ and contribute to increasing levels [35].

We confirmed known association of obesity with triglyceride and inverse association with HDL-C [36]. We observed an inverse correlation of the waist circumference and waist to height ratio, but not BMI, with apoA-I. Although height may influence the association, since it associates negatively with liver fat content but not with visceral fat mass [37], our observation supports the role of visceral fat in HDL metabolism in obesity and the concept that low HDL-C in obesity may be associated with apoA-I degradation and impaired cholesterol efflux from lipoprotein and cellular sources [38]. However, there is considerable heterogeneity 


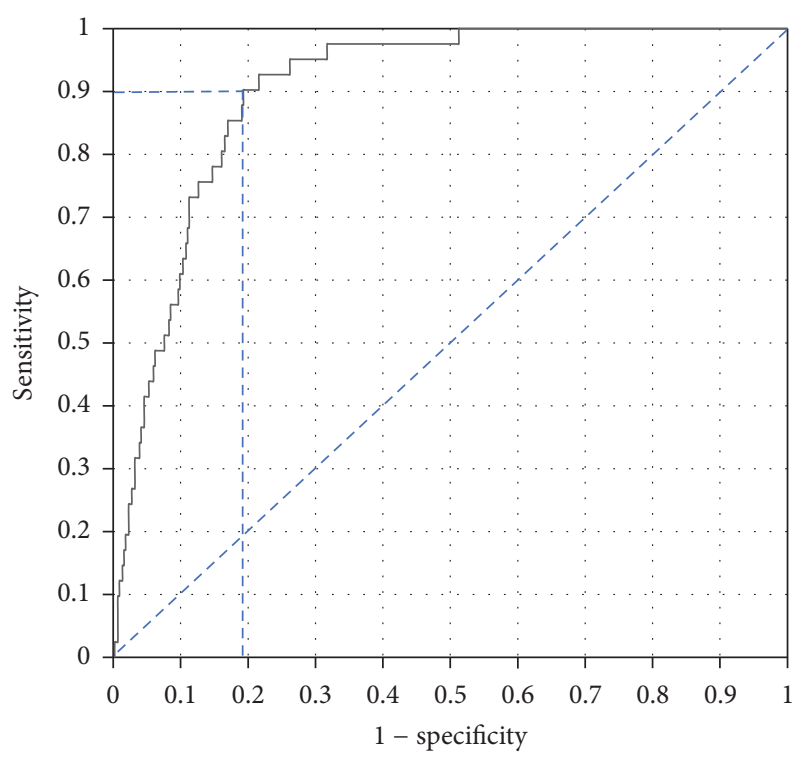

FIGURE 1: The receiver operating characteristic (ROC) curve from logistic regression selecting apoA-I and apoB to apoA-I ratio as identifying those with $\mathrm{MHO}$ among all obese individuals. The area under the ROC was 0.902 (that is, $C$-statistic $=0.902$ ) indicating high discrimination of the model.

in prediabetes phenotypes associated with obesity, and we did not evaluate non-alcoholic fatty liver disease (NAFLD) manifesting as increased hepatic fat. Furthermore, the presence of NAFLD could account for increased carotid intimamedia thickness (cIMT) and a more dysregulated lipid and apolipoprotein profile [39]. Also male participants were much more likely to have $\mathrm{MHO}$; men had a more than four times chance of being $\mathrm{MHO}$ than women. This finding is unique in obese Cherokee participants and different to sex differences for $\mathrm{MHO}$ among other populations that have favored females [40]. This observation can possibly be accounted for by differences in daily exercise or unknown factors [41]; however we have previously observed higher rates for the metabolic syndrome in 20-40-year-old males (30.9\%) than in females $(22.0 \%)$ consistent with higher overall risk for males [3].

Our findings emphasize the atherogenic role of low and dysfunctional HDL-C in obesity since low HDL-C is associated with risk for cardiovascular disease [42] even when LDL-C is lowered [43]. Both apoA-I and HDL-C are known to be low in obesity and the low levels are attributed to HDL's interaction with VLDL mediated by hepatic triglyceride lipase [44]. These metabolic events are in part attributed to insulin resistance [44] and account for our finding that $\mathrm{MHO}$ participants have less insulin resistance and higher apoA-I, possibly accounting for a less atherogenic effect, particularly when combined with lower apoB and the apoB:apoA-I ratio [45]. The high $C$-statistic and retention in the model support prediction of $\mathrm{MHO}$ and lower risk attributable to the apoB:apoA-I ratio $[45,46]$ and support use of the ratio as a measure of cardiovascular risk attributable to obesity.

\section{Conclusions}

Measurements of apoA-I, apoB, and apoC-III contained in HDL and non-HDL contribute information on risk and provide rationale for cholesterol, triglyceride, and apoCIII lowering strategies in obese individuals. Association of apoC-III in non-HDL, a known correlate of triglyceride, with insulin resistance and lipids supports early risk reduction treatments including lifestyle and lipid-lowering medications. Obese cases may present with relatively normal metabolic syndrome criteria and should be managed with caution since obese individuals may increase their risk for both atherosclerosis and T2D. ApoA-I was positively and apoB : apoA-I was negatively associated with the chance of being $\mathrm{MHO}$, and both were good identifiers for being $\mathrm{MHO}$ suggesting that these apolipoprotein measures can be helpful in assessing risk in obese individuals, especially in men who have a much higher chance of being MHO than women.

$\begin{array}{ll}\text { Abbreviations } & \\ \text { Apo: } & \text { Apolipoprotein before A-I, B, and } \\ & \text { C-III } \\ \text { BMI: } & \text { Body mass index } \\ \text { FPG: } & \text { Fasting blood glucose } \\ \text { TC: } & \text { Total cholesterol } \\ \text { TG: } & \text { Triglyceride } \\ \text { LDL-C: } & \text { LDL-cholesterol } \\ \text { HDL-C: } & \text { HDL-cholesterol } \\ \text { apoB: apoA-I ratio: } & \text { Apolipoprotein B to A-I ratio } \\ \text { LpA-1: A-II: } & \text { Lipoproteins containing apoA-I } \\ & \text { and apoA-II } \\ \text { SBP: } & \text { Systolic blood pressure } \\ \text { DBP: } & \text { Diastolic blood pressure } \\ \text { Log: } & \text { Logarithm } \\ \text { HOMA-IR: } & \text { Homeostasis index for insulin } \\ & \text { resistance } \\ \text { WHR: } & \text { Waist to height ratio } \\ \text { Waist: } & \text { Waist circumference } \\ \text { Non-HDL-apoC-III: } & \text { ApoC-III in non-HDL } \\ \text { HDL-apoC-III: } & \text { ApoC-III in HDL. } \\ & \end{array}$

\section{Ethical Approval}

The manuscript was approved for publication by the Cherokee Nation Institutional Review Board and the Indian Health Service.

\section{Disclosure}

The opinions expressed in this paper are those of the authors and do not necessarily reflect the views of the Indian Health Service.

\section{Conflicts of Interest}

The authors declare there are no conflicts of interest regarding publication of this paper. 


\section{Acknowledgments}

The authors wish to express their appreciation to the Cherokee people and to the health care and administrative officials of the Cherokee Nation in Tahlequah, Oklahoma, for their support and assistance. The authors also thank the administrators and staff of the Cherokee Nation clinics at Stilwell, Salina, Sallisaw, and Jay. The Cherokee Diabetes Study is supported by the National Institute of Diabetes and Digestive and Kidney Diseases, Bethesda, MD, by Grant R01 DK47920 awarded to Elisa T. Lee, Ph.D. The late Petar Alaupovic, Ph.D., Head of The Lipid and Lipoprotein Laboratory at the Oklahoma Medical Research Foundation provided laboratory support and initiated the hypothesis and analysis.

\section{References}

[1] R. H. Eckel, W. W. Barouch, and A. G. Ershow, "Report of the National Heart, Lung, and Blood Institute-National Institute of Diabetes and Digestive and Kidney Diseases working group on the pathophysiology of obesity-associated cardiovascular disease," Circulation, vol. 105, no. 24, pp. 2923-2928, 2002.

[2] B. V. Howard, E. T. Lee, L. D. Cowan et al., "Rising tide of cardiovascular disease in American Indians: The Strong Heart Study," Circulation, vol. 99, no. 18, pp. 2389-2395, 1999.

[3] P. Alaupovic, P. Blackett, W. Wang, and E. Lee, "Characterization of the metabolic syndrome by apolipoproteins in the Oklahoma Cherokee," Journal of the Cardiometabolic Syndrome, vol. 3, no. 4, pp. 193-199, 2008.

[4] S. M. Haffner, M. P. Stern, H. P. Hazuda, B. D. Mitchell, and J. K. Patterson, "Cardiovascular risk factors in confirmed prediabetic individuals. Does the clock for coronary heart disease start ticking before the onset of clinical diabetes?" The Journal of the American Medical Association, vol. 263, no. 21, pp. 2893-2898, 1990.

[5] J. B. Meigs, P. W. Wilson, C. S. Fox et al., "Body mass index, metabolic syndrome, and risk of type 2 diabetes or cardiovascular disease," The Journal of Clinical Endocrinology \& Metabolism, vol. 91, no. 8, pp. 2906-2912, 2006.

[6] N. Stefan, H. U. Häring, F. B. Hu, and M. B. Schulze, "Metabolically healthy obesity: epidemiology, mechanisms, and clinical implications," The Lancet Diabetes \& Endocrinology, vol. 1, no. 2, pp. 152-162, 2013.

[7] J. Elovson, J. E. Chatterton, G. T. Bell et al., "Plasma very low density lipoproteins contain a single molecule of apolipoprotein B," Journal of Lipid Research, vol. 29, no. 11, pp. 1461-1473, 1988.

[8] A. D. Sniderman, "Applying apoB to the diagnosis and therapy of the atherogenic dyslipoproteinemias: a clinical diagnostic algorithm," Current Opinion in Lipidology, vol. 15, no. 4, pp. 433438, 2004.

[9] P. R. Blackett, K. S. Blevins, M. Stoddart et al., "Body mass index and high-density lipoproteins in Cherokee Indian children and adolescents," Pediatric Research, vol. 58, no. 3, pp. 472-477, 2005.

[10] G. Walldus, I. Jungner, A. H. Aastveit, I. Holme, C. D. Furberg, and A. D. Sniderman, "The apoB/apoA-I ratio is better than the cholesterol ratios to estimate the balance between plasma proatherogenic and antiatherogenic lipoproteins and to predict coronary risk," Clinical Chemistry and Laboratory Medicine, vol. 42, no. 12, pp. 1355-1363, 2004.

[11] M. Burstein and R. Morlin, "Precipitation of serum lipoproteins by anionic detergents in the presence of bivalent cations," Revue
Européenne D'études Cliniques et Biologiques, vol. 15, no. 1, pp. 109-113, 1970.

[12] W. Wang, S. Khan, P. Blackett, P. Alaupovic, and E. Lee, "Apolipoproteins A-I, B, and C-III in young adult Cherokee with metabolic syndrome with or without type 2 diabetes," Journal of Clinical Lipidology, vol. 7, no. 1, pp. 38-42, 2013.

[13] C. O. Mendivil, E. B. Rimm, J. Furtado, S. E. Chiuve, and F. M. Sacks, "Low-density lipoproteins containing apolipoprotein CIII and the risk of coronary heart disease," Circulation, vol. 124, no. 19, pp. 2065-2072, 2011.

[14] F. M. Sacks, P. Alaupovic, L. A. Moye et al., "VLDL, apolipoproteins $\mathrm{B}, \mathrm{CIII}$, and $\mathrm{E}$, and risk of recurrent coronary events in the cholesterol and recurrent events (CARE) trial," Circulation, vol. 102, no. 16, pp. 1886-1892, 2000.

[15] D. H. Blankenhorn, R. H. Selzer, D. W. Crawford et al., "Beneficial effects of colestipol-niacin therapy on the common carotid artery. Two- and four-year reduction of intima-media thickness measured by ultrasound," Circulation, vol. 88 , no. 1, pp. 20-28, 1993.

[16] M. K. Jensen, E. B. Rimm, J. D. Furtado, and F. M. Sacks, "Apolipoprotein C-III as a potential modulator of the association between HDL-cholesterol and incident coronary heart disease," Journal of the American Heart Association, vol. 1, no. 2, Article ID e000232, 2012.

[17] The TG and HDL Working Group of the Exome Sequencing Project, National Heart, Lung, and Blood Institute, "Loss-offunction mutations in APOC3, triglycerides, and coronary disease," The New England Journal of Medicine, vol. 371, no. 1, pp. 22-31, 2014.

[18] M. J. Graham, R. G. Lee, T. A. Bell III et al., "Antisense oligonucleotide inhibition of apolipoprotein C-III reduces plasma triglycerides in rodents, nonhuman primates, and humans," Circulation Research, vol. 112, no. 11, pp. 1479-1490, 2013.

[19] M. C. Wyler Von Ballmoos, B. Haring, and F. M. Sacks, “The risk of cardiovascular events with increased apolipoprotein CIII: a systematic review and meta-analysis," Journal of Clinical Lipidology, vol. 9, no. 4, pp. 498-510, 2015.

[20] B. Talayero, L. Wang, J. Furtado, V. J. Carey, G. A. Bray, and F. M. Sacks, "Obesity favors apolipoprotein E- and C-III-containing high density lipoprotein subfractions associated with risk of heart disease," Journal of Lipid Research, vol. 55, no. 10, pp. 21672177, 2014.

[21] M. D. Curry, W. J. McConathy, J. D. Fesmire, and P. Alaupovic, "Quantitative determination of human apolipoprotein CcIII by electroimmunoassay," Biochimica et Biophysica Acta (BBA)_Lipids and Lipid Metabolism, vol. 617, no. 3, pp. 503-513, 1980.

[22] M. D. Curry, P. Alaupovic, and C. A. Suenram, "Determination of apolipoprotein A and its constitutive A-I and A-II polypeptides by separate electroimmunoassays," Clinical Chemistry, vol. 22, no. 3, pp. 315-322, 1976.

[23] M. D. Curry, A. Gustafson, P. Alaupovic, and W. J. McConathy, "Electroimmunoassay, radioimmunoassay, and radial immunodiffusion assay evaluated for quantification of human apolipoprotein B," Clinical Chemistry, vol. 24, no. 2, pp. 280-286, 1978.

[24] D. Samocha-Bonet, D. Chisholm, K. Tonks, L. Campbell, and J. Greenfield, "Insulin-sensitive obesity in humans-a "favorable fat" phenotype?" Trends in Endocrinology \& Metabolism, vol. 23, no. 3, pp. 116-124, 2012. 
[25] N. Kloting, M. Fasshauer, A. Dietrich et al., "Insulin-sensitive obesity," American Journal of Physiology. Endocrinology and Metabolism, vol. 299, no. 3, pp. E506-E515, 2010.

[26] N. Stefan, K. Kantartzis, J. Machann et al., "Identification and characterization of metabolically benign obesity in humans," Archives of Internal Medicine, vol. 168, no. 15, pp. 1609-1616, 2008.

[27] Z. Cui, K. P. Truesdale, P. T. Bradshaw, J. Cai, and J. Stevens, "Three-year weight change and cardiometabolic risk factors in obese and normal weight adults who are metabolically healthy: the atherosclerosis risk in communities study," International Journal of Obesity, vol. 39, no. 8, pp. 1203-1208, 2015.

[28] S. Eisenberg, J. R. Patsch, J. T. Sparrow, A. M. Gotto, and T. Olivecrona, "Very low density lipoprotein. Removal of apolipoproteins C-II and C-III-1 during lipolysis in vitro," The Journal of Biological Chemistry, vol. 254, no. 24, pp. 1260312608, 1979.

[29] K. Olin-Lewis, R. M. Krauss, M. La Belle et al., "ApoC-III content of apoB-containing lipoproteins is associated with binding to the vascular proteoglycan biglycan," Journal of Lipid Research, vol. 43, no. 11, pp. 1969-1977, 2002.

[30] C. Zheng, V. Azcutia, E. Aikawa et al., "Statins suppress apolipoprotein CIII-induced vascular endothelial cell activation and monocyte adhesion," European Heart Journal, vol. 34, no. 8, pp. 615-624, 2013.

[31] J. Altomonte, L. Cong, S. Harbaran et al., "Foxol mediates insulin action on apoC-III and triglyceride metabolism," The Journal of Clinical Investigation, vol. 114, no. 10, pp. 1493-1503, 2004.

[32] P. R. Blackett, K. S. Blevins, E. Quintana et al., “ApoC-III bound to apoB-containing lipoproteins increase with insulin resistance in Cherokee Indian youth," Metabolism: Clinical and Experimental, vol. 54, no. 2, pp. 180-187, 2005.

[33] V. Ceccarelli, G. Nocentini, C. Riccardi et al., "Effect of dietary saturated fatty acids on HNF- $4 \alpha$ DNA binding activity and ApoCIII mRNA in sedentary rat liver," Molecular and Cellular Biochemistry, vol. 347, no. 1-2, pp. 29-39, 2011.

[34] N. Faghihnia, L. M. Mangravite, S. Chiu, N. Bergeron, and R. M. Krauss, "Effects of dietary saturated fat on LDL subclasses and apolipoprotein CIII in men," European Journal of Clinical Nutrition, vol. 66, no. 11, pp. 1229-1233, 2012.

[35] S. Caron, A. Verrijken, I. Mertens et al., "Transcriptional activation of apolipoprotein CIII expression by glucose may contribute to diabetic dyslipidemia," Arteriosclerosis, Thrombosis, and Vascular Biology, vol. 31, no. 3, pp. 513-519, 2011.

[36] B. V. Howard, G. Ruotolo, and D. C. Robbins, "Obesity and dyslipidemia," Endocrinology and Metabolism Clinics of North America, vol. 32, no. 4, pp. 855-867, 2003.

[37] N. Stefan, H. Häring, F. B. Hu, and M. B. Schulze, "Divergent associations of height with cardiometabolic disease and cancer: epidemiology, pathophysiology, and global implications," The Lancet Diabetes \& Endocrinology, vol. 4, no. 5, pp. 457-467, 2016.

[38] G. F. Lewis and D. J. Rader, "New insights into the regulation of HDL metabolism and reverse cholesterol transport," Circulation Research, vol. 96, no. 12, pp. 1221-1232, 2005.

[39] N. Stefan, A. Fritsche, F. Schick, and H. Häring, "Phenotypes of prediabetes and stratification of cardiometabolic risk," The Lancet Diabetes \& Endocrinology, vol. 4, no. 9, pp. 789-798, 2016.

[40] C. M. Phillips, C. Dillon, J. M. Harrington et al., "Defining metabolically healthy obesity: role of dietary and lifestyle factors," PLoS ONE, vol. 8, no. 10, Article ID e76188, 2013.
[41] S. Velho, F. Paccaud, G. Waeber, P. Vollenweider, and P. Marques-Vidal, "Metabolically healthy obesity: different prevalences using different criteria," European Journal of Clinical Nutrition, vol. 64, no. 10, pp. 1043-1051, 2010.

[42] N. E. Miller, D. S. Thelle, O. H. Forde, and O. D. Mjos, "The Tromso heart-study. High-density lipoprotein and coronary heart-disease: a prospective case-control study," The Lancet, vol. 1, no. 8019, pp. 965-968, 1977.

[43] P. Barter, A. M. Gotto, J. C. LaRosa et al., "HDL cholesterol, very low levels of LDL cholesterol, and cardiovascular events," The New England Journal of Medicine, vol. 357, no. 13, pp. 1301-1310, 2007.

[44] B. Vergès, M. Adiels, J. Boren et al., "Interrelationships between the kinetics of VLDL subspecies and hdl catabolism in abdominal obesity: a multicenter tracer kinetic study," Journal of Clinical Endocrinology and Metabolism, vol. 99, no. 11, pp. 42814290, 2014.

[45] G. Walldius, I. Jungner, I. Holme, A. H. Aastveit, W. Kolar, and E. Steiner, "High apolipoprotein B, low apolipoprotein A-I, and improvement in the prediction of fatal myocardial infarction (AMORIS study): a prospective study," The Lancet, vol. 358, no. 9298, pp. 2026-2033, 2001.

[46] W. A. Van Der Steeg, S. M. Boekholdt, E. A. Stein et al., "Role of the apolipoprotein B-apolipoprotein A-I ratio in cardiovascular risk assessment: a case-control analysis in EPIC-Norfolk," Annals of Internal Medicine, vol. 146, no. 9, pp. 640-648, 2007. 

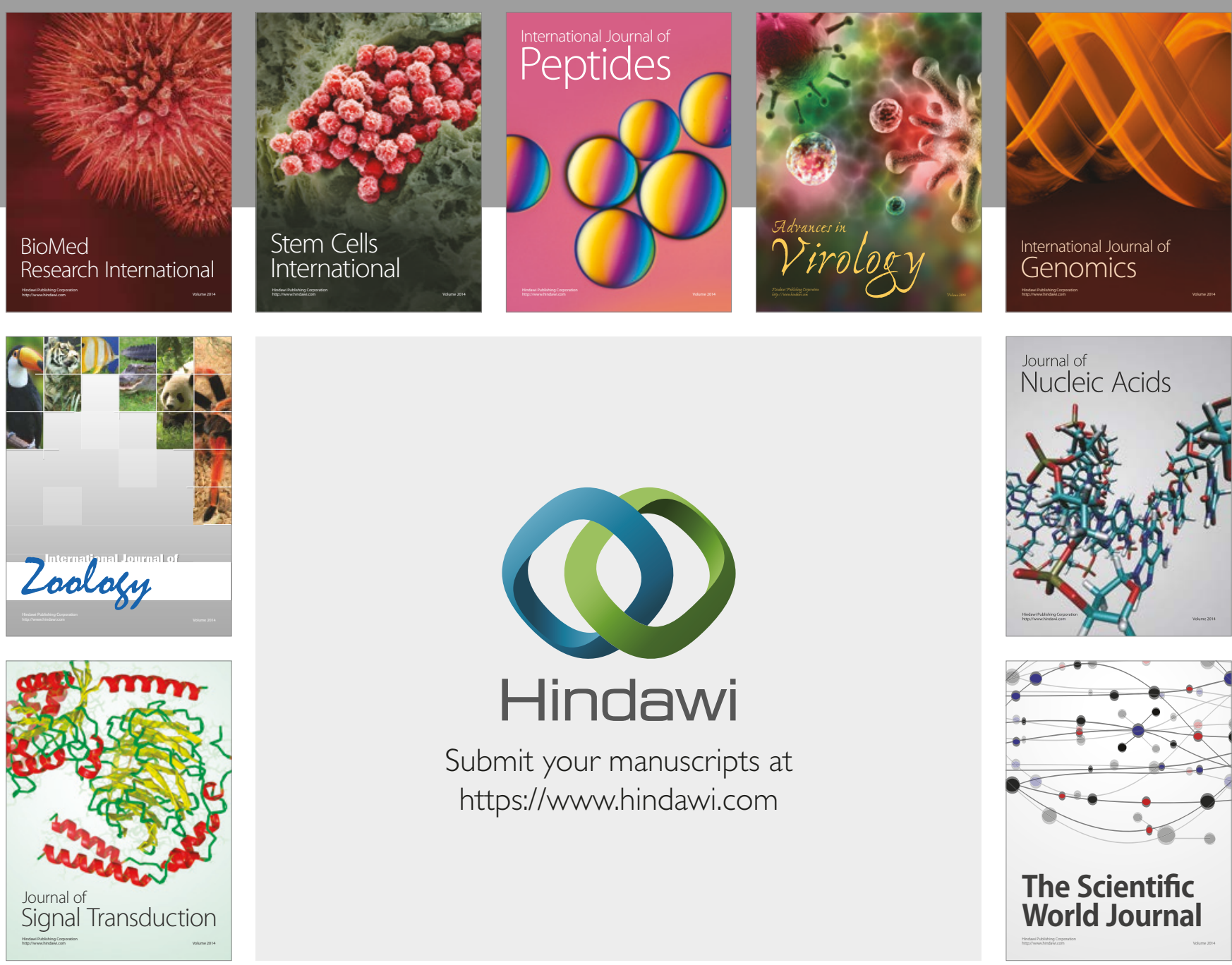

Submit your manuscripts at

https://www.hindawi.com
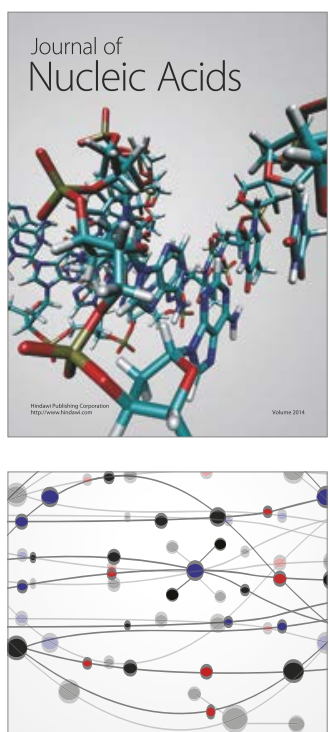

The Scientific World Journal

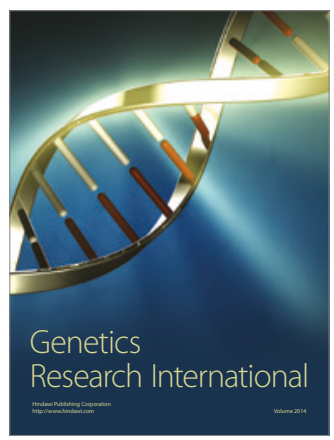

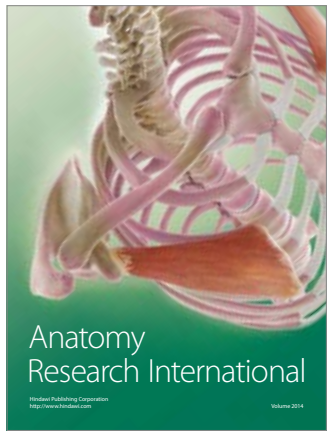

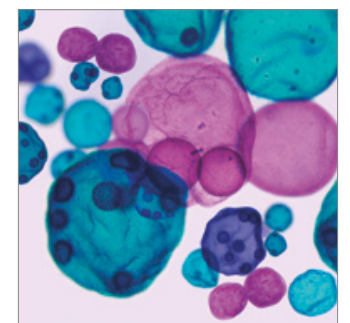

International Journal of Microbiology
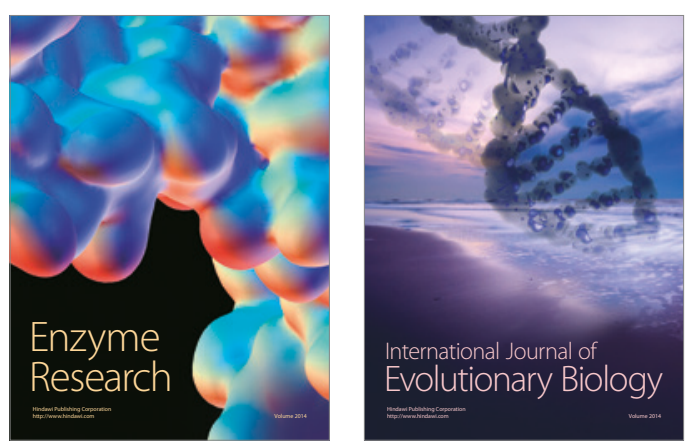
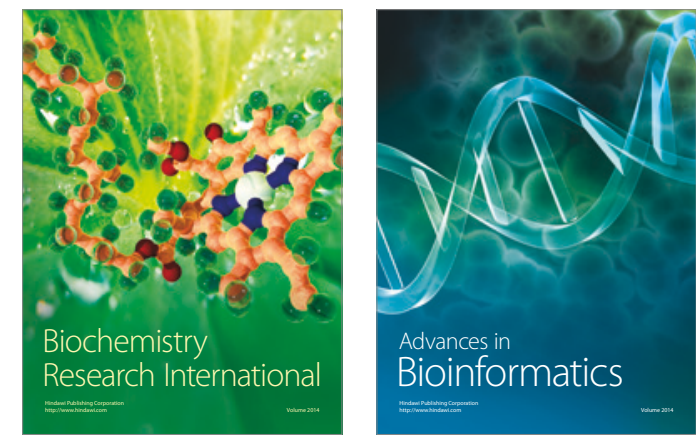

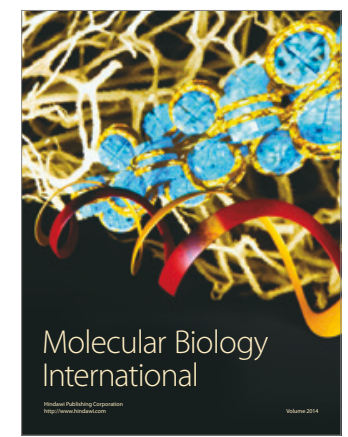

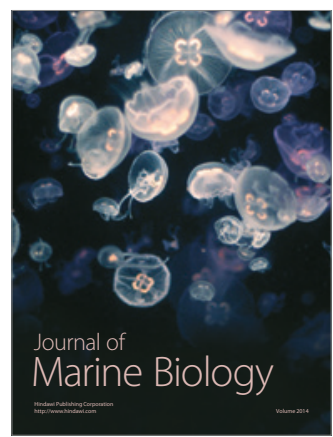

